\begin{tabular}{|c|c|c|}
\hline Case Reports in & \multicolumn{2}{|c|}{ Case Rep Gastroenterol 2015;9:113-119 } \\
\hline Gastroenterology & $\begin{array}{l}\text { DOI: } 10.1159 / 000382072 \\
\text { Publisnea onine:. April Z4, } 2015\end{array}$ & $\begin{array}{l}\text { (c) } 2015 \text { S. Karger AG, Basel } \\
1662-0631 / 15 / 0091-0113 \$ 39.50 / 0 \\
\text { www.karger.com/crg }\end{array}$ \\
\hline & \multicolumn{2}{|c|}{$\begin{array}{l}\text { This is an Open Access article licensed under the terms of the Creative Commons } \\
\text { Attribution-NonCommercial } 3.0 \text { Unported license (CC BY-NC) (www.karger.com/OA } \\
\text { license), applicable to the online version of the article only. Distribution permitted for non } \\
\text { commercial purposes only. }\end{array}$} \\
\hline
\end{tabular}
commercial purposes only.

\section{Prolonged Survival in a Case of Chemotherapy-Sensitive Gastric Cancer That Produced Alpha- Fetoprotein and Protein Induced by Vitamin K Antagonist-II}

\author{
Naotaka Ogasawara ${ }^{a}$ Emiko Takahashi ${ }^{b}$ Tomoko Matsumoto ${ }^{a}$ \\ Manami Amaike ${ }^{a}$ Mako Nohara $^{a}$ Kazuhiro Nagao ${ }^{a}$ Masahide Ebi ${ }^{a}$ \\ Yasushi Funaki $^{\mathrm{a}}$ Makoto Sasaki $^{\mathrm{a}}$ Kunio Kasugai ${ }^{\mathrm{a}}$ \\ Departments of ${ }^{\mathrm{a}}$ Gastroenterology and ${ }^{\mathrm{b}}$ Pathology, Aichi Medical University School of \\ Medicine, Nagakute, Japan
}

\section{Key Words}

Alpha-fetoprotein - Chemotherapy · Gastric cancer · Human epidermal growth factor receptor 2 . Protein induced by vitamin $\mathrm{K}$ antagonist-II

\begin{abstract}
The number of reported cases of alpha-fetoprotein (AFP)-producing gastric cancer has gradually increased, with a reported prevalence of $1.3-1.5 \%$ of all gastric cancer cases. However, reports of gastric cancer accompanied by elevated serum levels of both AFP and protein induced by vitamin $\mathrm{K}$ antagonist-II (PIVKA-II) are rare. The prognosis of AFP- and PIVKA-IIproducing gastric cancer has been reported to be very poor because the tumor cells were considered to have a high malignant potential and the cancer progressed rapidly. We described a case of gastric cancer producing AFP and PIVKA-II in which chemotherapy was effective and resulted in prolonged survival, and these two tumor markers were useful for monitoring the treatment response. Routine health screening using upper abdominal ultrasonography revealed hepatic tumors in an apparently healthy 65 -year-old man. Whole-body computed tomography (CT) revealed multiple hepatic tumors, and an esophagogastroduodenoscopy (EGD) revealed a Bormann type 3 tumor in the lower stomach. A biopsy specimen confirmed that the tumor was immunohistochemically positive for AFP, PIVKA-II, and human epidermal growth factor receptor 2. After chemotherapy, the gastric tumor appeared as a
\end{abstract}

KARGER 125:s $\quad \begin{aligned} & \text { Naotaka Ogasawara, MD } \\ & \text { Department of Gastroenterology } \\ & \text { Aichi Medical University School of Medicine } \\ & 1-1 \text { Yazakokarimata, Nagakute, Aichi 480-1195 (Japan) } \\ & \text { E-Mail nogasa@aichi-med-u.ac.jp }\end{aligned}$


Ogasawara et al.: Prolonged Survival in a Case of Chemotherapy-Sensitive Gastric Cancer That Produced Alpha-Fetoprotein and PIVKA-II

small elevated lesion on EGD, and CT revealed a remarkable reduction in the size of the metastatic liver tumors. The patient is still alive, 35 months after the initial chemotherapy.

(C) 2015 S. Karger AG, Basel

\section{Introduction}

Protein induced by vitamin K antagonist-II (PIVKA-II) and alpha-fetoprotein (AFP) are considered to be specific tumor markers of hepatocellular carcinoma, although PIVKA-II is considered the more specific of the two [1]. Since Kudo et al. [2] first reported a case of PIVKA-II- and AFP-producing gastric cancer, a number of similar cases have been described. The number of reported cases of AFP-producing gastric cancer has also gradually increased [3], with a reported prevalence of $1.3-1.5 \%$ of all gastric cancer cases [3, 4]. However, reports of gastric cancer accompanied by elevated serum levels of both AFP and PIVKA-II are rare [2], and a previous review article described only 16 such cases [5]. The prognosis of AFP- and PIVKA-II-producing gastric cancer has been reported to be very poor because the tumor cells were considered to have a high malignant potential and the cancer progressed rapidly. In addition, most of these cancers metastasized and were drug resistant. A recently developed oral derivative of 5 -fluorouracil, $\mathrm{S}-1$, has been found to be effective in approximately $45 \%$ of advanced gastric cancer cases $[6,7]$, and it has also proved effect in some cases of AFP-producing gastric cancer [8].

Here, we describe a case of gastric cancer producing AFP and PIVKA-II, which proved to be useful markers of therapeutic response. This cancer was sensitive to chemotherapy, which resulted in prolonged survival.

\section{Case Report}

A routine health screening using upper abdominal ultrasonography revealed hepatic tumors in an apparently healthy 65-year-old man with no known family history of gastrointestinal disorders or malignant diseases. He had no abdominal discomfort or other symptoms and no lymphadenopathy or thyroid swelling, and his abdominal findings were normal. The laboratory analysis revealed elevated serum levels of aspartate transaminase (AST) at $46 \mathrm{IU} / \mathrm{l}$, an alkaline phosphatase (ALP) level of $510 \mathrm{IU} / \mathrm{l}$, and a lactate dehydrogenase (LDH) level of 1,474 IU/l. Other laboratory findings upon admission were within normal limits. Tests for hepatitis $\mathrm{C}$ virus antibody (HCV-Ab) and hepatitis B virus surface antigen (HBs- $\mathrm{Ag}$ ) were negative. As for tumor markers, serum AFP and PIVKA-II levels were both extremely high at 88,329 ng/ml (AFP-L3, 75.8\%) and $296 \mathrm{mAU} / \mathrm{ml}$, respectively, whereas serum levels of carcinoembryonic antigen (CEA) and carbohydrate antigen 19-9 (CA19-9) were in the normal range. Whole-body computed tomography (CT) revealed multiple hepatic tumors, which were less dense than normal liver tissue. The enhanced CT using contrast agent showed that the tumors had peripheral ring enhancement and a heterogeneous interior (fig. 1a, b). CT also revealed a swollen lymph node surrounding the lesser curve of the stomach (fig. 1b, arrowhead) and a thickening of the stomach wall (fig. 1c, arrowheads). The hepatic tumors had a low intensity on diffusion-weighted magnetic resonance imaging (MRI), and enhanced MRI with contrast agent showed that the periphery of the hepatic tumors was enhanced in the arterial phase (fig. 1d), but the hepatic tumors were not enhanced in the hepatobiliary phase (fig. 1e). CT and MRI findings suggested that the hepatic tumors were metastatic, and fine needle aspiration biopsy of these lesions revealed them to be poorly 
Ogasawara et al.: Prolonged Survival in a Case of Chemotherapy-Sensitive Gastric Cancer That Produced Alpha-Fetoprotein and PIVKA-II

differentiated adenocarcinomas and thus metastases from the gastrointestinal cancer. An esophagogastroduodenoscopy (EGD) revealed a Bormann type 3 advanced tumor about $30 \mathrm{~mm}$ in diameter in the lower part of the stomach (fig. 2a, b). A biopsy specimen showed that the tumor was a poorly differentiated adenocarcinoma (fig. 3a), corresponding with the specimen obtained from the fine needle aspiration biopsy of the hepatic tumors. Immunohistochemical evaluation of a tumor biopsy specimen revealed that the tumor cells were positive for AFP (fig. 3b) and PIVKA-II (fig. 3c), as well as human epidermal growth factor receptor 2 (HER2; fig. 3d). Based on the TNM classification (International Union Against Cancer, UICC), this AFP- and PIVKA-II-producing gastric cancer with hepatic metastasis was diagnosed as stage IV (T2N1M1). S-1 (80 mg/m² on days 1-14), cisplatin (CDDP; $60 \mathrm{mg} / \mathrm{m}^{2}$ on day 1$)$, and trastuzumab ( $8 \mathrm{mg} / \mathrm{kg}$ on day 1 ) were administered every 3 weeks for 15 cycles. After 15 cycles of chemotherapy, AFP and PIVKA-II serum levels were greatly reduced, at $826.5 \mathrm{ng} / \mathrm{ml}$ and $51 \mathrm{mAU} / \mathrm{ml}$, respectively. EGD performed 8 months after chemotherapy showed a remarkable reduction in the size of the tumor, which appeared as an excavated lesion with marginal protrusion (fig. 2c). After 15 cycles of chemotherapy with S-1, CDDP, and trastuzumab, the regimen was changed due to renal dysfunction, and instead S-1 (60 mg/m² on days $1-14)$, CPT-11 (150 mg/m $\mathrm{m}^{2}$ on day 1 , to replace CDDP), and trastuzumab $(8 \mathrm{mg} / \mathrm{kg}$ on day 1$)$ were administered every 3 weeks for 15 cycles. After 30 cycles of chemotherapy, AFP and PIVKA-II serum levels were both lower, at $10.1 \mathrm{ng} / \mathrm{ml}$ and $41 \mathrm{mAU} / \mathrm{ml}$, respectively. EGD performed 25 months after chemotherapy revealed a more remarkable reduction in the size of the tumor, which appeared as a small elevated lesion with scar (fig. 2d), and CT findings at the same time showed a remarkable reduction in the size of the metastatic liver tumors defined as partial response according to the revised response evaluation criteria in solid tumors (RECIST) guideline (version 1.1; fig. 1f). The patient is still alive at the time of reporting, 35 months after the initial chemotherapy, without an increase in the level of serum tumor markers, and continues to be administered chemotherapy with a combination of S-1, CPT-11, and trastuzumab.

\section{Discussion}

We describe a case of advanced gastric cancer with liver metastasis accompanied by elevated levels of serum AFP and PIVKA-II, in which prolonged survival was achieved due to effective combination chemotherapy. Immunohistochemical examination demonstrated that the gastric cancer cells were positive for both AFP and PIVKA-II, thereby allowing the diagnoses of an AFP- and PIVKA-II-producing gastric cancer. Gastric cancers rarely produce PIVKA-II, and only 16 such cases were identified in a recent review [5]. Consequently, its actual prevalence remains uncertain. In many cases, gastric biopsy specimens appear histologically as poorly or moderately differentiated adenocarcinoma; however, a hepatoid pattern has also been observed during the examination of surgically excised or autopsy samples [5]. In 6 of the 9 previously reported cases of AFP- and PIVKA-II-producing gastric cancers, the tumor was found to be poorly differentiated adenocarcinoma, whereas 4 showed hepatoid differentiation in biopsy specimens [9]. Histologically, the tumor in this case was a poorly differentiated adenocarcinoma without hepatoid differentiation, and this type of gastric cancer actually produced AFP and PIVKA-II.

AFP-producing gastric cancers are associated with a poor prognosis, even when diagnosed at an early stage, and frequently give rise to hepatic metastases [10]. Chemotherapy for gastric cancer is the main treatment option for patients with a liver metastasis, but systemic chemotherapy with a combination of anti-cancer drugs such as CDDP, 5-fluorouracil, 
Ogasawara et al.: Prolonged Survival in a Case of Chemotherapy-Sensitive Gastric Cancer That Produced Alpha-Fetoprotein and PIVKA-II

and CPT-11 generally has poor efficacy. Although S-1, a novel oral derivative of 5-fluorouracil, has been found to be effective in several cases of AFP-producing gastric cancers, longterm survival with S-1 therapy has not been reported for PIVKA-II-producing gastric cancers [11]. The prognosis in these cases largely depends on whether liver metastases are present. According to a review of 16 cases of AFP- and PIVKA-II-producing gastric cancers [5], all 3 patients without liver metastasis were still alive and free of recurrence 9, 15, and 17 months after surgery. Twelve of the 13 patients with liver metastasis died from their disease, and the longest survival period of those patients was 6 months. The follow-up period for the only patient who was still alive with liver metastasis was just 2 months [5]. Recent studies have shown that several combination chemotherapies significantly improved the survival of patients with advanced gastric cancer [12]. The SPIRITS trial (S-1 plus CDDP vs. S-1 in a randomized controlled trial for the treatment of advanced gastric cancers) showed that combined therapy with S-1 and CDDP significantly prolonged survival when given as a first-line treatment for advanced gastric cancers. The overall survival (OS) of patients treated with S-1 plus CDDP was 13.0 months compared with 11.0 months for patients treated with S-1 alone [13]. Moreover, the administration of HER2-targeted therapies, in particular trastuzumab combined with chemotherapy for metastatic HER2-positive gastric cancers, resulted in improved response rates, time to progression, and OS. Trastuzumab is a molecularly targeted agent approved as a standard treatment in gastric cancers [14,15]. This agent induces antibody-dependent cellular cytotoxicity, inhibits HER2-mediated signaling, and prevents the extracellular domain of HER2 from being cleaved. The Trastuzumab for Gastric Cancer (ToGA) study was an open-label phase III, randomized controlled trial undertaken in 122 centers among 24 countries [14]. It revealed that the addition of trastuzumab to conventional cytotoxic chemotherapy was clinically beneficial compared to chemotherapy alone in terms of tumor response, which suggested that combined chemotherapy with trastuzumab could be a novel, acceptable option for patients with HER2-positive advanced gastric cancer. Trastuzumab also prolonged the survival of patients with HER2-overexpressing gastric cancers [14]. In the case we describe here, the gastric cancer was diagnosed as stage IV, and the patient underwent chemotherapy instead of surgical treatment. The gastric cancer cells were positive for HER2 on immunohistochemistry, and the patient was administered combination chemotherapy consisting of S-1, CDDP, and trastuzumab, following the practice described in previously reported cases $[14,15]$. After 15 cycles of combination chemotherapy, the patient in our case suffered renal dysfunction and was consequently administered S-1, CPT-11 (to replace CDDP), and trastuzumab, but has now survived for 35 months after the initial chemotherapy and continues to have low tumor markers levels. He continues to receive S-1, CPT-11, and trastuzumab combination treatment. We think that this chemotherapy regimen, and in particular the use of trastuzumab, contributed to the prolonged survival of our patient, despite the generally poor prognosis associated with gastric cancers that produce AFP. To our knowledge, there was no report of AFP-producing gastric cancer accompanying the expression of HER2.

We described a case of gastric cancer producing AFP and PIVKA-II in which chemotherapy was effective and resulted in prolonged survival, and these 2 tumor markers were useful for monitoring the treatment response.

\section{Disclosure Statement}

The authors declare no conflicts of interest. 
Ogasawara et al.: Prolonged Survival in a Case of Chemotherapy-Sensitive Gastric Cancer That Produced Alpha-Fetoprotein and PIVKA-II

\section{References}

1 Fujiyama S, Morishita T, Hashiguchi O, et al: Plasma abnormal prothrombin (des-gamma-carboxy prothrombin) as a marker of hepatocellular carcinoma. Cancer 1988;61:1621-1628.

$\checkmark 2$ Kudo M, Takamine Y, Nakamura K, et al: Des-gamma-carboxy prothrombin (PIVKA-II) and alphafetoprotein-producing IIc-type early gastric cancer. Am J Gastroenterol 1992;87:1859-1862.

-3 Chang YC, Nagasue N, Abe S, et al: Comparison between the clinicopathologic features of AFP-positive and AFP-negative gastric cancers. Am J Gastroenterol 1992;87:321-325.

4 Chang YC, Nagasue N, Abe S, et al: Alpha fetoprotein producing early gastric cancer with liver metastasis: report of three cases. Gut 1991;32:542-545.

-5 Takahashi Y, Inoue T, Fukusato T: Protein induced by vitamin K absence or antagonist II-producing gastric cancer. World J Gastrointest Pathophysiol 2010;1:129-136.

-6 Koizumi W, Kurihara M, Nakano S, et al: Phase II study of S-1, a novel oral derivative of 5-fluorouracil, in advanced gastric cancer. For the S-1 Cooperative Gastric Cancer Study Group. Oncology 2000;58:191-197.

7 Osugi H, Takada N, Takemura M, et al: Oral fluoropyrimidine anticancer drug TS-1 for gastric cancer patients with peritoneal dissemination. Oncol Rep 2002;9:811-815.

-8 Fujita H, Yoshioka I, Inokuchi M, et al: A patient with advanced gastric cancer in the gastric tube whose QOL was improved by TS-1 (in Japanese). Gan To Kagaku Ryoho 2002;29:443-447.

-9 Takano S, Honda I, Watanabe S, et al: PIVKA-II-producing advanced gastric cancer. Int J Clin Oncol 2004;9: 330-333.

10 Uefuji K, Ichikura T, Tamakuma S: Roles of histological findings and serum AFP levels in the prognosis of AFP-producing gastric cancers. Jpn J Clin Oncol 1994;24:135-140.

11 Sohda T, Tomioka Y, Inomata S, et al: Alpha-fetoprotein (AFP)- and des-gamma-carboxy prothrombin (DCP)-producing adenocarcinoma of the stomach with liver metastasis in a patient with chronic hepatitis $\mathrm{C}$. Intern Med 2005;44:294-298.

$\$ 12$ Murad AM, Santiago FF, Petroianu A, et al: Modified therapy with 5-fluorouracil, doxorubicin, and methotrexate in advanced gastric cancer. Cancer 1993;72:37-41.

13 Koizumi W, Narahara H, Hara T, et al: S-1 plus cisplatin versus S-1 alone for first-line treatment of advanced gastric cancer (SPIRITS trial): a phase III trial. Lancet Oncol 2008;9:215-221.

14 Bang YJ, Van Cutsem E, Feyereislova A, et al: Trastuzumab in combination with chemotherapy versus chemotherapy alone for treatment of HER2-positive advanced gastric or gastro-oesophageal junction cancer (ToGA): A phase 3, open-label, randomised controlled trial. Lancet 2010;376:687-697.

$\checkmark 15$ Roukos DH: Targeting gastric cancer with trastuzumab: new clinical practice and innovative developments to overcome resistance. Ann Surg Oncol 2010;17:14-17. 


\section{Case Reports in \\ Gastroenterology}

\begin{tabular}{l|l}
\hline \multicolumn{2}{l|}{ Case Rep Gastroenterol 2015;9:113-119 } \\
\hline DOI: $10.1159 / 000382072$ & $\begin{array}{l}\text { C } 2015 \text { S. Karger AG, Basel } \\
\text { www.karger.com/crg }\end{array}$ \\
\hline
\end{tabular}

Ogasawara et al.: Prolonged Survival in a Case of Chemotherapy-Sensitive Gastric Cancer That Produced Alpha-Fetoprotein and PIVKA-II
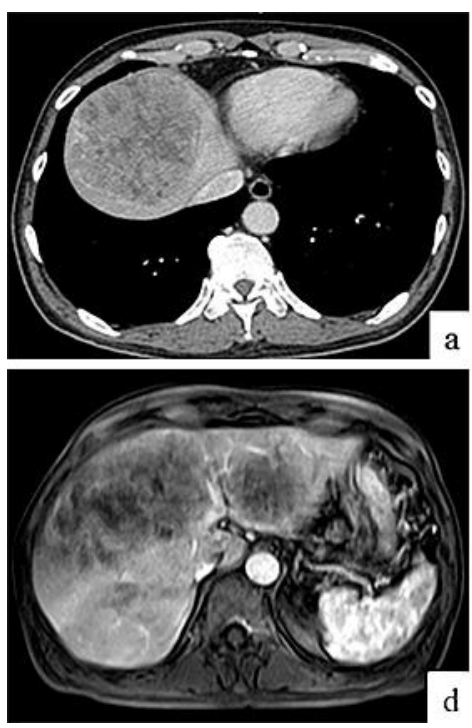
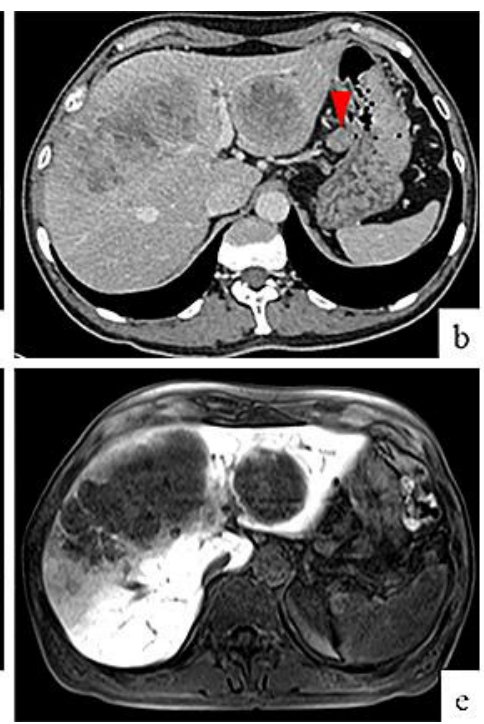
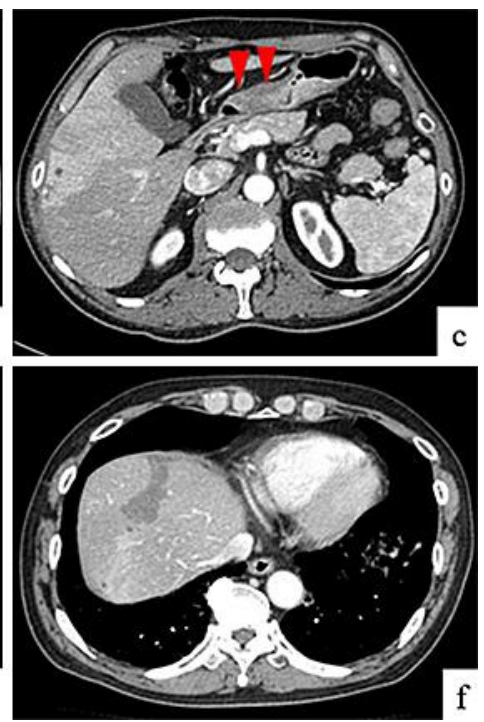

Fig. 1. CT on admission and 25 months after chemotherapy. Enhanced CT on admission revealed ring enhancement around the periphery of the tumors and heterogeneous enhancement within the tumors (a). CT also revealed multiple hepatic tumors with heterogeneous enhancement, and a swollen lymph node surrounding the lesser curve of the stomach (b, arrowhead). Thickening of the stomach wall was indicative of a gastric tumor that was thought to have invaded the muscularis propria (c, arrowheads). MRI on admission showed enhancement at the periphery of the tumors and heterogeneous enhancement within the tumors in the arterial phase (d), but there was no enhancement during the delayed phase (e). CT finding 25 months after chemotherapy showed a remarkable reduction in the size of the metastatic liver tumors without enhancement (f). 
Ogasawara et al.: Prolonged Survival in a Case of Chemotherapy-Sensitive Gastric Cancer That Produced Alpha-Fetoprotein and PIVKA-II
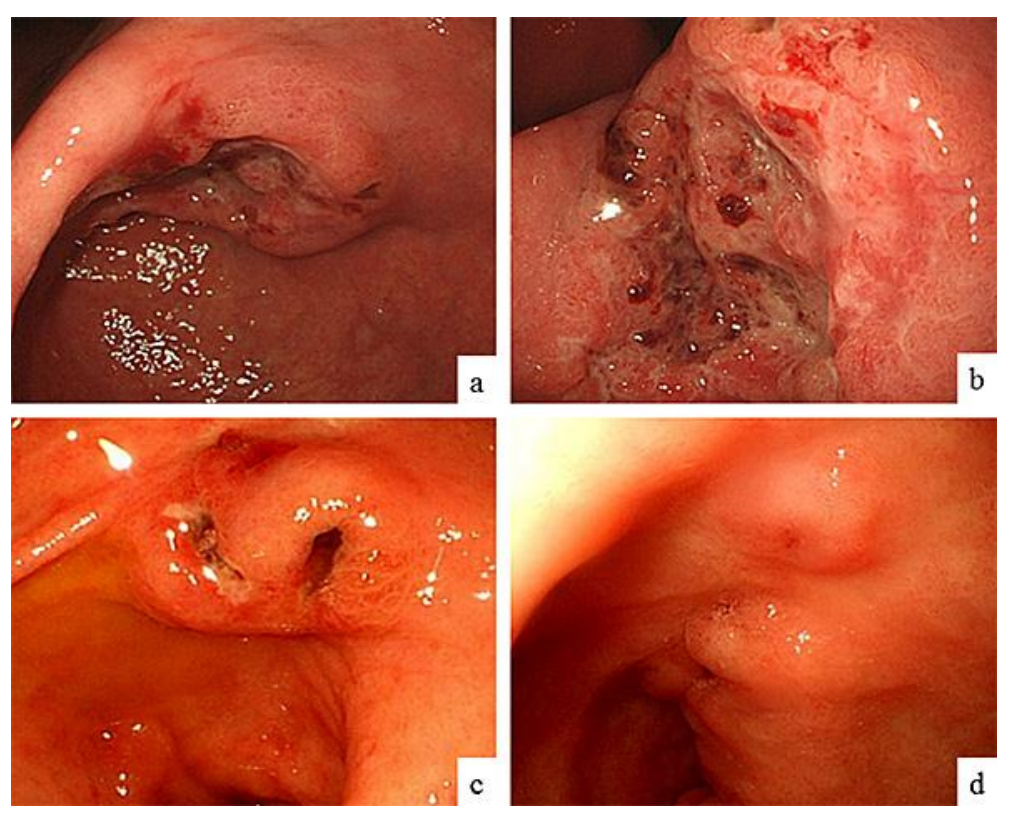

Fig. 2. Endoscopic findings before chemotherapy and 25 months after chemotherapy. EGD before chemotherapy revealed a Bormann type 3 advanced tumor about $30 \mathrm{~mm}$ in diameter in the lower part of the stomach (a). Closer view of the gastric tumor (b). EGD performed 8 months after chemotherapy showed a remarkable reduction in the size of the tumor, which had the appearance of an excavated lesion with marginal protrusion (c). EGD performed 25 months after chemotherapy revealed an even greater reduction in the size of the tumor, which had the appearance of an extremely small elevated lesion with a scar (d).
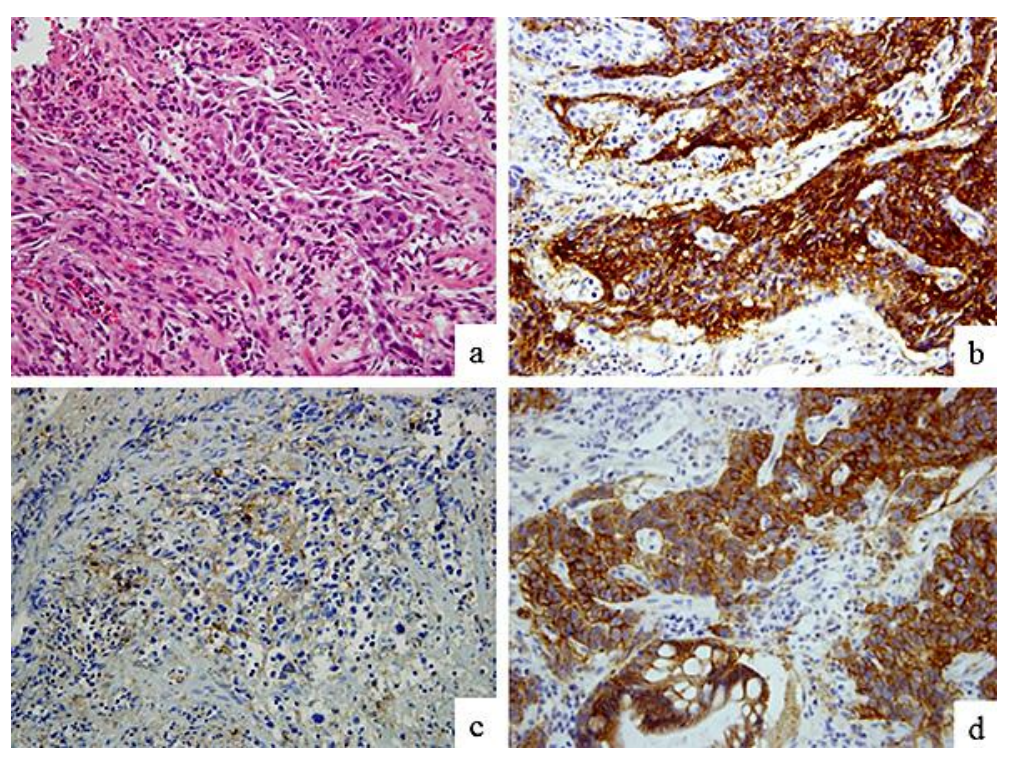

Fig. 3. Hematoxylin and eosin (HE) staining and immunohistochemical findings of the gastric tumor biopsy specimen. HE staining revealed that the tumor was a poorly differentiated adenocarcinoma (a). Immunohistochemical evaluation of a tumor biopsy specimen revealed that the tumor cells were positive for AFP (b), PIVKA-II (c), and HER2 (d). Original magnification $\times 400(a-d)$. 